\title{
Mapping the health systems response to violence against women: $\mathrm{A}$ comparative analysis of five LMIC settings (2015-2020)
}

\author{
Shegufta Shefa Sikder \\ Care \\ Rakhi Ghoshal \\ Care India \\ Padma Deosthali \\ Care India \\ Chandni Jaishwal \\ Emory University \\ Nobhojit Roy ( $\square$ nobhojit.roy@ki.se) \\ Karolinska Institute
}

\section{Research Article}

Keywords: violence against women, gender-based violence, health system response, one-stop centers, low- and middle-income countries

Posted Date: February 23rd, 2021

DOI: https://doi.org/10.21203/rs.3.rs-200380/v1

License: (c) (1) This work is licensed under a Creative Commons Attribution 4.0 International License. Read Full License 


\section{Abstract \\ Background}

Violence against women (VAW) is prevalent globally, and the health sector is a key entry point for survivors to receive care. However, documentation on the rollout of health system response to violence against women is lacking in low and middle-income countries. This paper maps the operationalization of health systems response to violence against women in five low- and middle-income countries (LMIC) to identify core learnings.

\section{Methods}

We selected five LMIC contexts that were actively addressing national-level health system response from 2015 to 2020 and where we had access to practitioners directly engaged in national rollout. We synthesized publicly available data and program reports according to the components of the Health Systems Framework to Address VAW.

\section{Results}

One-stop centers were found to be the dominant model of care located in hospitals implemented by the health systems in four countries except Brazil, where one-stop centers are not located in hospitals and do not provide health services. While each setting has implemented in-service training as key to addressing provider knowledge, attitudes and practice, significant gaps remain in addressing frequent staff turnover, provision of training at scale, and documentation on the impact of training. Health system protocols for VAW address sexual violence but do not uniformly include clinical and health policy responses for other forms of violence. In Brazil, Nepal, and Sri Lanka, harmful practices such as virginity testing remain within clinical protocols. Providing privacy to survivors within health facilities was a universal challenge.

\section{Conclusions}

Significant efforts have been made to address provider attitudes towards provision of care and in protocolized delivery of care to survivors, primarily through one-stop centers. Improvements can be made in data collection on training impact on provider attitudes and practices, in provider identification of VAW survivors, and in prioritization of VAW within health system budgeting, staffing, and political priorities. Beyond hospital-based one-stop centers at tertiary and district levels, primary health facilities need to provide first-line support for survivors to avoid delays in response to VAW as well as for secondary prevention.

\section{Background}

Globally one in three women experience violence in her lifetime. For many women the violence is perpetrated by an intimate partner or family member [1]. The prevalence of intimate partner violence ranges from 15 percent in Japan to 71 percent in Ethiopia [2, 3]. Such violence has far-ranging reproductive, sexual, and mental health consequences [3]. Survivors access health facilities for medical treatment and care of the injuries and symptoms[4]. Thus, health providers are uniquely positioned to provide support to survivors, even beyond the immediate medical care [5]. However, support beyond the initial clinical response has not been successfully integrated into health care systems [6].

In 2014, the World Health Assembly stated that the health system is a key entry point for survivors to receive help and discussed a commitment for a health response as well as a framework for country strategies [7]. Based on the World Health Organization (WHO) health systems building blocks [8], a framework for a health systems response to survivors was developed in 2015 and adopted by the WHO [5]. The systems-level components that support health providers include training and skill development, development of a health information database, monitoring and evaluation, and setting in place of protocols and referrals chains; these are further supported through appropriate budgeting and a commensurate leadership at the top as well as coordination with other sectors and community engagement. Health systems have a critical role in addressing VAW within a multi-sectoral approach [5].

Despite these initial frameworks, additional limitations remain in health systems response for VAW. Typical challenges survivors face at health facilities include insensitive approach of providers, re-traumatization of her experience of violence, and victim-blaming [9-10]. Consequently, many survivors are forced to return to the place of abuse without any safety plan or support in place [11]. Cultural beliefs normalizing VAW reinforce the lack of support for survivors and exacerbate the challenges of proper health care delivery to survivors. Other challenges to providing a health system response include inadequate infrastructure and lack of coordination between the health sector and other support sectors. Several governments have begun to focus on putting in place structural support system for survivors. $\mathrm{H}$ systems interventions include implementation of appropriate policies and guidelines, focused budgeting, standardising protocols, and integration of a gender-sensitive response at the facility level [5]. The 2015 Lancet Series on VAW discussed the evidence base on addressing VAW and the role of the health sector, highlighting the need for evidence on addressing VAW in low- and middle-income countries (LMICs) [12]. In addition, in 2013 the WHO released its Policy and Clinical Guidelines for Response to Intimate Partner Violence based on systematic reviews drawing from evidence from largely highincome settings, emphasizing the gaps in evidence from LMICs [13].

Against this context of challenges in LMICs, and the significantly high prevalence of domestic and intimate partner violence therein [1, 6], this paper aims to study how LMIC health systems have operationalized health systems responses to VAW between 2015 and 2020 . The paper uses the health systems framework as the lens to interrogate each of its components for five specific LMIC settings. We identify the strengths and gaps in each setting and synthesizes the findings and highlight interventions which have contributed to health system addressal of violence against women (VAW).Using a comparative analysis, we conclude with a list of core learnings and recommendations relevant for other LMIC settings. 


\section{Methods}

We purposively selected LMIC countries that had recent or active health system programming (after 2015) on a national level as a response to VAW and where we had access to practitioners directly engaged in national rollout of these responses. To define the health systems rollout of VAW in LMICs, we utilized data from Nepal, Bangladesh, Sri Lanka, Brazil, and Rwanda (Table 1).

Data sources included: (i) data from publicly available large-scale surveys and (ii) program reports, reports by ministries and by non-governmental organizations, and reports identified by program practitioners. The information obtained from (i) and (ii) was synthesized as analytical summaries according to the components of the Health Systems Framework to Address VAW [5].

Table 1

Selected Country Contexts and Focus Issues

\begin{tabular}{|c|c|c|c|}
\hline Country & Focus & Health System Organization & $\begin{array}{l}\text { Human } \\
\text { Development } \\
\text { Index } \\
\text { Ranking } \\
(2020)\end{array}$ \\
\hline Bangladesh & $\begin{array}{l}\text { Multi-sectoral programme on Violence against } \\
\text { women (including one-stop centers and } \\
\text { separate initiative to integrate VAW into NGO } \\
\text { Service Delivery Project }\end{array}$ & $\begin{array}{l}\text { Bangladesh has a pluralistic unregulated healthcare system, with } \\
\text { government, for-profit private sector, not-for-profit private sector (mainly } \\
\text { the nongovernmental organizations), and the international development } \\
\text { organizations as actors }\end{array}$ & $133 / 190$ \\
\hline Brazil & $\begin{array}{l}\text { Houses for Women and addressing VAW at } \\
\text { primary health care levels }\end{array}$ & $\begin{array}{l}\text { Universal and free public health care is provided to all Brazilians as a } \\
\text { constitutional right within the Unified Health System. }\end{array}$ & $92 / 190$ \\
\hline Nepal & $\begin{array}{l}\text { National scale up of Health sector response to } \\
\text { VAW and across one-stop centers }\end{array}$ & $\begin{array}{l}\text { Health care services in Nepal are provided by both public and private } \\
\text { sectors with Govt spending of US\$ } 2.30 \text { per person and } 70 \% \text { of health } \\
\text { expenditure is out-of-pocket contributions. }\end{array}$ & $142 / 190$ \\
\hline Rwanda & $\begin{array}{l}\text { Scale up of quality assurance across one-stop } \\
\text { centers and application of quality assurance } \\
\text { standards }\end{array}$ & $\begin{array}{l}\text { Rwanda follows a universal health care model, through a community- } \\
\text { based health insurance scheme }\end{array}$ & $160 / 190$ \\
\hline Sri Lanka & $\begin{array}{l}\text { National upscaling of Mithuru Piyasa one-stop } \\
\text { center model }\end{array}$ & $\begin{array}{l}\text { Sri Lanka has a universal health care system through public (general) } \\
\text { hospitals and free outpatient departments }\end{array}$ & $72 / 190$ \\
\hline
\end{tabular}

\section{Results}

Results are presented by components of the Health System Framework to Address VAW [5]. Adapted to the WHO health systems building blocks [8], the Health Systems Framework on Violence Against Women identifies components within each health systems building block which constitute a health systems response [5]. Figure 1: Strengths and Gaps by Component of Health Systems Framework on Violence Against Women. VAW = violence against women; NGO = non-governmental organization.

\section{1a.Health providers supporting survivors}

Regarding health provider attitudes towards sexual and domestic violence in these settings, a 2019 review of one-stop centers (OSCs) found staff engaged in victim-blaming [22]. Though Brazil criminalizes domestic violence, many health providers are "still reluctant to engage with the issue [23]." A previous study among health providers in Rwanda showed universal agreement with the statement, "A man who rapes a woman cannot control his behaviour [21]," indicating normalization with the belief that men are not capable of controlling sexual urges.

\section{1b. Responding to survivors}

The first step of a survivor-centered response is identification. The WHO recommends identification through questions based on the presenting conditions and history, a process known as "clinical enquiry [13]." Identification of violence based on presenting signs and symptoms differs from "routine enquiry," in which clients are universally screened for violence, which is not recommended outside of antenatal care and mental health services as universal screening can overwhelm health systems $[5,13]$. In addition to identification of violence, the 2013 WHO guidelines delineate the role of health systems in provision of women-centered clinical response and first-line support, indicating that referring women to care is insufficient, but that health facilities have a responsibility to provide first-line sensitive support for survivors of VAW.

Within 12 health facilities of Rwanda,routine enquiry for VAW was integrated into family planning and antenatal counseling, even though provider training did not include gender sensitization and key aspects of women-centered care [28]. Project staff reported that disclosure rates of violence were extremely low (1-2 percent in the first six months) of this routine enquiry pilot [28]. Providers reported feeling overstretched and overburdened, while client feedback indicated that providers were inquiring about violence insensitively. These challenges precluded the expansion of routine enquiry.

In Bangladesh, nongovernmental organization (NGO) clinics implemented a routine screening pilot for identification of violence among women seeking health services. The NGO Health Service Delivery Project (NHSDP) was a health service delivery project in Bangladesh funded by the United States Agency for International Development. This project supported the delivery of reproductive, maternal, and child health services through a network of rural and urban local 
NGO clinics that targeted poor and underserved women of reproductive age [25]. The NHSDP trained its staff on VAW, implemented a routine screening pilot, and developed a national protocol on health response to VAW.

For cases of sexual assault, protocols in Brazil emphasize the responsibility of nurses for identifying survivors and referring them to health providers and appropriate institutions; the protocols do not specify the role of other health providers other than nurses. In Nepal and Sri Lanka, responsibilities of health care providers include identification, confidentiality, and informed consent, provision of first aid and LIVES, history taking, medical management, medico legal services, referral, and follow up [29-30]. In Sri Lanka, the public health midwife is tasked with identifying issues related to VAW and referring and connecting survivors to the health system. Specific to sexual violence, medical officers are identified as responsible for medical management and response [30].

\section{Health systems supporting health providers}

\section{2a. VAW in policy and implementation: leadership and governance}

Government prioritization of resources and interventions is essential to changing practices. All settings have included VAW in their health policy and programmes. Even where the country's social welfare departments are anchoring services (as in Bangladesh and Brazil), the role of health systems is delineated. However, these programs are not backed with directions from Ministries of Health on the role of each health facility. None of the settings provide legal sanction to domestic violence or rape, and in Bangladesh and Sri Lanka marital rape is not criminalized [32].

\section{2b. Financing}

Policy gets translated to practice through appropriate financing. Allocation of funds are required for in-service training of providers, for appointment of trained staff for running OSCs, and necessary infrastructure. The funds for OSCs come from government ministries of health in Nepal, Sri Lanka, and Rwanda. In Bangladesh, the majority of the funding from OSCs comes from international donors (including the Danish government for the Multi-Sectoral Programme on VAW) [33]. In Sri Lanka the OSC program is financed by the Ministry of Health, while international donors are only in a supportive role. The OSCs in Rwanda were initially co-funded by the United Nations and the government. Local level prioritization is well placed in Nepal where each Village Development Committee allocates 15 percent of its budget for women's issues, the microplanning for which takes place at the Health Facility Management Committee. Funds are available to reimburse the healthcare and legal expenses incurred by survivors, even though complex regulations, and administrative hoops challenge access [34]. In Brazil, funding allocations for VAW are decided are administered at the local levels within states.

\section{2c. Health infrastructure}

Providing privacy and confidentiality, availability of medicines and other supplies constitute infrastructure. In all the settings, data showed that providing privacy to the survivor emerged as a challenge. Additional challenges in all five settings included lack of furniture, information and communication material, instruments, and medicines, use of space and equipment designated for OSCs by other departments, and excessive patient load leading to overcrowding. In Nepal, OSCs were ranked as providing better privacy and confidentiality than health facilities, and this was attributed to the protocolized nature of VAW response at the OSCs. Ascore card used to rank OSCs in Nepal showed 82 percent of assessed OSCs operating with adequate space and equipment or supplies [27]. In all other settings, infrastructural challenges included lack of adequate health provider training besides the lack of space to ensure privacy.

\section{2d. Health workforce training/development}

Stakeholder training is built into the implementation plans in all contexts, with varying results. Frequent staff turnover remains a challenge to capacity building on VAW response. Health provider training programs in Nepal focus on improving competency and skills; nurses are mandated to identify survivors and doctors to provide medical treatment and to report medico-legal cases. Nepal addressed staffing gaps in its 2016 revised OSC Operational Guideline by appointing a minimum of one medical doctor and two to three staff nurses the OSC or as on call for 24-hour provision of service. A 2020 report on Nepal's OSCs indicates the majority addressed these staffing changes to be operational 24 hours a day [27]. Following training on health systems response, providers noted a new sense of responsibility they felt to support the survivor get justice, while noting that doctors not being relieved from their regular duty if they have to attend a court hearing at some hospitals remains a challenge [27]. The United Nations Population Fund (UNFPA) and NGO partners in Bangladesh support the government in providing staff training; they have developed a screening checklist, referral flow-chart, counselling guideline and provided training to the providers for essential services in their clinic networks [25]. A monthly review meeting with health providers, outreach workers, and their supervisors is conducted. The National Protocol for Health Sector Response to VAW in Bangladesh requires health providers to provide psychological first aid to survivors, medico legal examination and proper documentation. Sri Lanka focuses on training and capacity building too, both for health providers and the public health midwife. Sri Lanka introduced VAW as a module for public health midwives focused on knowledge-building, skill development for addressing and preventing VAW in the community, as well as on positive provider-client interaction and communication [30]. Since 2015 the government in Rwanda has focused on strengthening and scaling its OSC model in all provinces, improving documentation, implementing quality assurance standards, providing specialized support to children and adolescent survivors, training providers on first-line support for survivors and post-GBV health care, making appropriate referrals, and strengthening referral pathways, with help from its non-governmental partners.

In Sri Lanka, of all health providers who are trained in responding to VAW, one person gets designated as the nodal person, and s/he is given an additional four-day training on responding to VAW survivors. The nodal person works across the ministries of health and of women affairs. In Nepal the OSCs have designated staff for managing/responding to cases that come, and usually a nurse or paramedic and a psychosocial counsellor [34]. Information on the process of designating a nodal person for managing VAW cases from the other three contexts is vague.

While all countries reported that they train in-service providers, there was no documentation on impact of training on provider knowledge, attitudes, or practices. In Brazil, the Ministry of Health assembled an optional online curriculum for medical professionals on interpersonal and intimate partner violence [24]. However, till date, this training has been completed by less than 10 percent of the professionals, indicating a lack of uptake. In Bangladesh, in addition to 
initial training, the NGO Health Service Delivery Project integrated discussion on provider attitudes on VAW into monthly meetings between health staff and supervisors besides refresher trainings [25]. In Nepal, a 2016 study indicated a lack of training and knowledge around VAW among health providers [26]. While impact data on provider change in attitudes resulting from training on VAW were not available, staff nurses who received psychosocial counselling training noted how they have changed the language they use with survivors and have become aware of how to show sensitivity, respect, and empathy to survivors [27]. Nepal's Ministry of Health and Population introduced an in-service for medical doctors on autopsy and clinical medico-legal training, although training has not been initiated in recent years.

\section{2e. Health information system}

Documentation and information management in Nepal includes VAW data recorded at the national level on two different platforms, from all health facilities through the Health Management Information System (HMIS), and from the OSCs routed to the Prime Minister's Office. The VAW data is disaggregated along sex, age, type of violence, caste, ethnicity and disability. Additional indicators note the services provided to survivors and referrals made. Rwanda and Nepal are the only countries in this study which report data on VAW through HMIS. Since 2012, Rwanda has worked on streamlining its quality of health data which is collected from the community health workers and health facilities to HMIS and has integrated facility-level as well as household-level data. The nationalized database in Bangladesh is not yet functional; however, its Bureau of Statistics collects household level survey data on VAW once every four years [33]. A call center database accumulates the data on calls made by survivors documenting forms of assault, and referrals. The centralized system in Brazil records cases of VAW, though it was implied to the authors that the data is not reliable enough. Nonetheless, the reporting at the state level in Brazil is better managed than at the federal level. Sri Lanka does not capture data on medico-legal cases nor reports through HMIS.

\section{2f. Service delivery}

Service delivery for VAW includes psychosocial support, medical treatment, multi-sectoral referral including within health [13]. A One-Stop Center (OSC) provides multi-sectoral case management for survivors, including health, welfare, counselling, and legal services under one roof with the intention of minimizing referrals and the need to have survivors repeat their account [22]. Descriptions of the OSCs in each setting as well as available data on coverage and service quality are provided in Additional File 1.

OSCs were found to be the most dominant model of care implemented by the health systems in four countries (Nepal, Bangladesh, Sri Lanka, and Rwanda) in which OSCs are set up in hospitals. Brazil is the exception, with OSCs managed as separate centers providing services to survivors (judicial services, specialized police for assistance to VAW survivors, shelter assistance, psychosocial support, and economic livelihoods support) which do not include medical support. While Sri Lanka and Rwanda's OSCs were set up by the health system, the OSCs in Bangladesh and Nepal are set up as large multi-sectoral programmes with clear health sector roles and responsibilities.

In all settings, OSCs are present in hospitals within all districts within the country, with the exception of Brazil, in which OSCs called Houses for Women are at the regional level and are not hospital-based. Staff at OSCs are designated and trained in protocols detailing response to VAW. Referrals to OSCs come largely from police and directly from communities (Additional File 1). OSCs have created space for provision of counseling to survivors of violence in the hospital. GBV protocols specify that hospital departments, especially emergency and specialized departments, should refer clients for additional medical services at OSCs. However, as seen in Additional File 1, internal hospital referrals are not captured as a source of referral to OSCs. Gaps remain in training of health providers in identification of signs and symptoms of VAW.

\section{2g. Coordination and community engagement}

Coordination to facilitate internal and other sectoral referrals and community engagement are important axes of the Health Systems Framework to Address VAW. OSCs in Nepal have protocolized multisectoral coordination such as with the legal, judicial, and livelihoods. The referral pattern of OSC survivors shows community awareness about services as a portion of survivors reach OSCs directly [27]. In Sri Lanka's 2016 Demographic and Health Survey, 13 percent of surveyed women were aware of Mithuru Piyasa services [20]. In Sri Lanka since the Mithuru Piyasa centers are located within the hospital, intra-facility referrals are relatively smooth, though other-sectoral referrals are not formalized. Recognising the importance of engaging the community, Sri Lanka prioritized the use of information education communication material, with public health midwives responsible for community engagement. Given their access to the community, they remain critical for identifying survivors, especially those women who otherwise would continue to bear the violence in silence [35].

In the rest of the settings, structured intersectoral coordination and referral are lacking. Changes within the federal system in Nepal have resulted in uneven funding of multi-sectoral services for VAW survivors, such as safe homes and rehabilitation services [36]. The NGO Jhpiego oriented select community health volunteers in Nepal for identification and referral of survivors, showing that with appropriate training and safety mechanisms, community health workers can raise community awareness about VAW, facilitate support for survivors, and help prevent harmful practices [37]. In Bangladesh, while the Multi-Sectoral Programme on VAW does not have an explicit community engagement component, the initiative runs TV ads and radio spots for public awareness on the availability of VAW services [33]. The world's largest NGO (Building Resource Across Communities, or BRAC), as well as other NGOs, have implemented programs focusing on improving community engagement and awareness of VAW, though these services are not directly linked to the Multi-Sectoral Programme on VAW [38]. In Brazil, networks of psychologists and social workers, activists, and women-led organizations increase awareness on VAW, though efforts are not formally linked to available OSC services. Rwanda's Protocol on Treatment of Survivors of VAW demonstrates a model of inter-sectoral coordination for VAW and includes provisions for safety planning and community resources for survivors, though formal coordination with other sectors for VAW support is not protocolized [39].

\section{Discussion}

\section{Health providers supporting survivors}


These data indicate provider reluctance to respond to VAW, acceptance and justification of VAW, andlack of skills to respond. The lack of skills among providers to respond to VAW may be due to the fact that no country has included VAW in pre-service curriculum. The settings discussed also show that training, skill development, refresher orientations to counter norms on acceptability of VAW has the power to impact provider attitude, seen particularly in the case of Nepal. Health providers are part of the same society, and the attitude of acceptance, as well as of blaming the survivor for much of the violence, prevails $[40,41]$. In the five settings, VAW is normalized in society, as evidenced by Demographic and Health Survey and national survey indicators on attitudes on wife beating and prevalence of VAW (Table 2) [14-20].

Table 2

Prevalence of VAW and attitudes on wife-beating in selected LMICs

\begin{tabular}{|lll|}
\hline Country & $\begin{array}{l}\text { Percentage of women 15-49 reporting physical or sexual violence } \\
\text { committed by husband/partner }\end{array}$ & $\begin{array}{l}\text { Percentage of women who agree that wife-beating is } \\
\text { justified for any reason }\end{array}$ \\
\hline Bangladesh & 53 [14] & 28 [15] \\
\hline Nepal [16] & 28 & 29 \\
\hline $\begin{array}{l}\text { Rwanda } \\
{[17]}\end{array}$ & 34 & 41 \\
\hline Brazil [18] & 17 & 30 \\
\hline Sri Lanka & 17 [19] & 53 [20] \\
\hline Source: Demographic and Health Surveys and national survey indicators \\
\hline
\end{tabular}

\section{Responding to survivors}

As seen across these five settings, health system protocols for VAW specify response to sexual violence but do not uniformly include clinical and health policy responses for other forms of violence (such as emotional or economic violence). In Brazil, Nepal, and Sri Lanka, harmful practices such as virginity testing and assessment of hymen for sexual habituation remain within clinical protocols despite supportive policies on health system response to VAW. The two-finger test for checking presence of hymen remains within clinical protocols in Bangladesh despite a Supreme Court ban [32]. In addition to these traumatic practices, insensitive attitudes from health care providers among survivors have been documented [40-41].

\section{Leadership and governance}

Recognition of VAW in health policy and programme was crucial in rolling out a health system response. It translated into allocation of funds, training of providers and setting up of services within health facilities. As seen in Brazil, the change in political leadership which is not supportive of gender issues has adversely affected the programming on VAW [46].

\section{Health workforce training/development}

While each setting has acknowledged ongoing training as key to changing attitudes and building health systems response, challenges with in-service training include lack of availability of staff and frequent turnover. As conducted in Bangladesh, regular review meetings including discussion on VAW response with providers, outreach workers, and their supervisors was important to ongoing prioritization of health response. Short, targeted, in-service, simulation-based learning activities, spaced over time and reinforced with structured, ongoing practice sessions (known as low-dose, high-frequency trainings) were found to be less time-intensive than traditional in-service trainings and resulted in provider retention of key content [42, 43].

The structure in Sri Lanka whereby of all providers trained in VAW, one is designated nodal and is given additional training, is worthy of mention, reflecting the investment of the government in the issue [30]. Further, to have the person work across ministries is a progressive move, since this would facilitate collaboration and coordination across ministries, and thus sectors.

\section{Service delivery}

The OSC model is a significant commonality across the five settings; in Sri Lanka, Bangladesh, Nepal and Rwanda, they are located within the health facility, which works in favour of facilitation of medical support for the survivors, in Brazil OSCs are not part of the health sector [44]. Survivors coming to 0SC receive a standard of care according to protocols, although identification of VAW by providers remains poor.

In most countries the number of OSCs have increased over time. Also, OSCs in all settings were able to ensure better privacy than the facilities in corresponding settings [22]. Recruitment and retention of staff emerged as an issue across every setting. In Rwanda, practitioners suggested staggering staff rotations in the future to prevent service stoppages for VAW response, particularly for forensic evidence collection [28]. While OSCs have resulted in provision of care for VAW at tertiary and district levels, OSC evaluations call for an adapted model of OSCs, in which tertiary and regional hospitals have specialized OSC units for comprehensive VAW response services, and lower level facilities provide first-line support with referral for specialized services as needed [33].

Recognising the need for first-line support of survivors at the primary healthcare levels prior to external referral [45], a multi-country initiative, Healthcare Responding to Violence and Abuse (HERA) is underway in Brazil, Nepal, Sri Lanka, and the occupied Palestinian territories. The HERA programme has developed and is piloting clinician training and referral interventions in primary care sexual and reproductive services initiative is designed to evaluate the differentials in the effectiveness of two approaches: between (a) training a small specialized group to identify survivors and provide first-line support, and (b) 
training all health workers at the primary level. Outcomes of interest include disclosure, first-line support and referral. Scheduled for completion in 2021, this mixed method research programme will report on the feasibility and acceptability of those interventions in terms of is qualitatively exploring longitudinal changes in beliefs and actions of trained health providers on identification, response, and referral and has already reported on readiness of primary care systems to respond to violence against women [46-47].

\section{Societal Context embedding Health Systems}

Within the five selected countries, Rwanda ranks the highest for gender equality (\#9 globally on the Global Gender Index [53]), followed by Bangladesh (\#50), Brazil (\#92), and Nepal and Sri Lanka (\#101 and \#102 respectively) [48, 49]. Of the five case studies, Brazil is the only middle-income country (with 2018 Gross National Income (GNI) per capita of $\$ \mathbf{1 4 , 0 6 8 )}$ ) and Sri Lanka as lower middle income (GNI per capita at $\$ 11,611)$. Even though Brazil is noted as having attained gender parity in health and survival, Brazil ranks the highest for gender inequality in the Latin America region (22nd out of 25 countries in Latin America). In sub-Saharan Africa, Rwanda is confirmed as the best performer in Sub-Saharan Africa due to its high proportion of female representation in government and health gains. Despite these gains, two-thirds of surveyed women indicate acceptability of wife-beating, indicating high levels of normalization of violence despite institutional gains [18]. Bangladesh is the highest performer in the South Asia region in the Global Gender Index due to gains in gender parity in health and survival and female representation in parliament [49]. These data indicate the enabling environment within the health system and political structures more broadly for gender equality, which affect implementation of all programs including response to VAW.

\section{Key Summaries}

This table summarizes the key findings from the contexts. Nepal's health system response to VAW represents the most lessons learned on operationalizing health systems components, including 24-hour operationalization of OSCs, appointment of designated staff for VAW response, provider training on sensitivity and clinical response, VAW data reporting through HMIS and centrally, legal sanction and protocol for health system response to VAW, and community engagement for identification and referral of services. Recent changes in the federal system leave uneven funding of multi-sectoral services for VAW survivors, such as safe homes and rehabilitation services [27, 36].

\begin{tabular}{|c|c|}
\hline Nepal & Bangladesh \\
\hline $\begin{array}{l}\text { The OSCs are in all districts, offering a full range of support services, with } \\
\text { designated trained staff nurses. Service delivery at the OSC is protocolized. } \\
\text { Has strong governance with the Prime Minister's Office directly involved in } \\
\text { monitoring VAW data. Prioritization at the community level is borne out by } \\
\text { the } 15 \text { percent allocation of funds for awareness and response issues. } \\
\text { Infrastructure is a challenge, which in turn affects privacy. Training of } \\
\text { providers and evaluation takes place in an ongoing basis. }\end{array}$ & $\begin{array}{l}\text { The OSCs are linked with regional trauma centers, indicating a strategic } \\
\text { understanding of the nuances of VAW and its health consequences. } \\
\text { Separate from OSCs, NGO-led implementation of screening checklist and } \\
\text { referral flow-chart facilitate identification and service provision at health } \\
\text { clinics. The Directorate General of Health and Ministry of Women and } \\
\text { Children's Affairs work together to provide VAW response. Infrastructure } \\
\text { constraints and privacy challenges remain within the facility setting. A } \\
\text { bulk of the financing is by foreign donors. }\end{array}$ \\
\hline Sri Lanka & Rwanda \\
\hline $\begin{array}{l}\text { Sri Lanka provides OSC services in all districts, with Public Health Midwives } \\
\text { focused on empowering survivors as well as responding to the community. } \\
\text { Laws are in place and with foreign donors moving to the margins, it is } \\
\text { appreciable that the government is willing to take on the financing of the } \\
\text { response to the VAW agenda. A nodal person is designated who is given } \\
\text { additional training on VAW, and who has the authority to work across } \\
\text { ministries is worth mentioning. Two weak links are intersectoral referral } \\
\text { network and lack of documentation of cases. }\end{array}$ & $\begin{array}{l}\text { The OSC model exists in all districts though it is weak on intersectoral } \\
\text { coordination. Has policies and data capturing processes in place, but } \\
\text { documentation is poor, suggesting inadequate monitoring. Substantial } \\
\text { financial support is provided from foreign donors with minimized } \\
\text { government fund prioritization for VAW response. Despite ranking high on } \\
\text { global gender indices, health provider attitudes continue to normalize VAW } \\
\text { and challenges in infrastructure prohibit privacy. }\end{array}$ \\
\hline \multicolumn{2}{|l|}{ Brazil } \\
\hline
\end{tabular}

\section{Top Lessons Learned}

1. There is an absence of pre-service training of health providers on sensitivity to gender and VAW as well as identification of signs and symptoms of VAW.

2. In-service training should be ongoing, with a need for integrating VAW training in medical and nursing education. Measurement of the impact of training on KAP is essential to assess change.

3. The location of one-stop centers within the hospital provides an opportunity for early identification of violence based on clinical enquiry by trained providers. Closer monitoring of data, including documentation on follow-up of survivors, service, and referral data, would help inform health system response to VAW. Linking the OSCs with regional trauma centers shall ensure quicker identification of VAW cases that reach the trauma dept owing to severe physical injury.

4. Links to community structures and awareness of VAW services have been established in all settings, underlining its relevance across all contexts.

5. Designating a nodal person who is given the power to work across ministries can foster strong intersectoral coordination and implement best practices such as regular case reviews.

\section{Strengths and Limitations}


This paper compares the uptake of the Health Systems framework of building a health systems response across five LMIC settings. This paper synthesizes a range of evidence to identify the core dimensions of a successful VAW response, showing what has worked and what has not, over a period of five years in five settings. This contextual understanding would strongly facilitate further program and intervention plans for other LMIC settings in developing the response.

The authors identify two limitations of this paper: (i) the selection of the five settings was purposive and based on access to material which would help provide the comparative analysis; (ii) the category of evidence is not homogeneous across settings despite the authors' efforts. Some of the literature is from the grey domain (such as program reports, annual reports) since the published literature examining health systems response is limited in the peer-reviewed academic domain.

\section{Conclusion}

These case studies provide compelling examples of how LMICs have operationalized health system response to VAW. Significant efforts have been made to address provider attitudes towards provision of sensitive care and in protocolized delivery of care and support to survivors, primarily through one-stop centers. However, improvements can be made in data collection on the impact of training on provider knowledge attitudes and practices, in provider identification of VAW through signs and symptoms, and in prioritization of VAW within the health system and political priorities. Even in countries which have hospital-based OSCs at tertiary and district levels, lower level health facilities need to provide first-line support for survivors to avoid delays in response to VAW as well as for secondary prevention.

\section{List Of Abbreviations}

\section{BRAC}

Building Resource Across Communities

GNI

gross national income

HERA

Healthcare Responding to Violence and Abuse

HMIS

Health Management Information System

LIVES

Listen, Inquire about Needs and Concerns, Validate, Enhance Safety, and Support to Information and Services

LMIC

low- and middle- income country

NGO

Nongovernmental Organization

NHSDP

Nongovernmental Organization Health Service Delivery Project

OSC

one-stop center

UNFPA

The United Nations Population Fund

VAW

violence against women

WHO

World Health Organization

\section{Declarations}

We confirm that all methods were carried out in accordance with relevant guidelines and regulations.

\section{Ethics approval and consent to participate}

This research utilized publicly available secondary data and program reports. No research was conducted with human subjects.

\section{Consent for publication}

Not applicable as this manuscript does not contain any individual person's data in any form (including any individual details, images or videos).

\section{Availability of data and materials}

Not applicable to this article as no datasets were generated or analysed during the current study. A summary table synthesizing findings by the Health Systems Framework for Response to Violence Against Women is available from the corresponding author upon request.

\section{Competing interests}


We declare no financial or non-financial competing interests.

\section{Funding}

No external funding sources were applied for this manuscript.

\section{Authors' contributions}

SS and CJ synthesized available information by components of the Health Systems Framework for Response to Violence Against Women. SS and RG drafted the manuscript text. PD and NR edited all sections of the manuscript, contributed to manuscript sections, and provided key input.

\section{Acknowledgements}

We acknowledge the contribution of Dr. Ana Flávia d'Oliveira, Daniel Lima, Shamsi Kazimbaya, Joya Banerjee, Dr. Abul Hossain, Lovely Yeasmin Jeba, Dr. Halida Akhter, Hashitha Abeywardana, Poonam Rishal, and Dr. Roshni Amatya for identification of program reports on health system response to VAW in their contexts. We thank Dr. Kavitha Ranganathan, Dr. Anita Gadgil, Dr. Dell Saulnier, and Dr. Gene Feder for their review and inputs to this manuscript.

\section{References}

1. World Health Organization. Global and regional estimates of violence against women: prevalence and health effects of intimate partner violence and nonpartner sexual violence.

https://apps.who.int/iris/bitstream/handle/10665/85239/9789241564625_eng.pdf;jsessionid=DD0647DAF7B5FBE5629B1D1A46615AE6?sequence=1 (2013). Accessed Dec 11, 2020.

2. World Health Organization. WHO Multi-country Study on Women's Health and Domestic Violence against Women Initial results on prevalence, health outcomes and women's responses. https://www.google.com/url?

sa $=t \& r c t=j \& q=\& e s r c=s \&$ source=web\&cd=\&ved=2ahUKEwi9xYDcq8btAhVEGMOKHQwPCecQFjABegQIARAC\&url=http\%3A\%2F\%2Fwhqlibdoc.who.int\%2Fpu (2005). Accessed Dec 11, 2020.

3. Dhar D, McDougal L, Hay K, et al. Associations between intimate partner violence and reproductive and maternal health outcomes in Bihar, India: a crosssectional study. Reprod Health. 2018;109(7) https://doi.org/10.1186/s12978-018-0551-2.

4. Bhate-Deosthali P, Rege S, Pal P, Nandi S, Bhatla N, \& Kashyap A. Role of the Health Sector in Addressing Intimate Partner Violence in India. International Centre for Research on Women. https://www.icrw.org/wp-content/uploads/2018/11/Role-of-the-Health-Sector-in-Addressing-IPV-in-India_rev.pdf (2018). Accessed Dec 11, 2020.

5. Garcia-Moreno C, Hegarty K, d'Olivera AFL, Koziol-Maclain J, Colombini M, Feder G. The health-systems response to violence against women. Lancet. 2015;385 (9977): 1567-1579. http://dx.doi.org/10.1016/S0140-6736(14)61837-7.

6. Ellsberg M, Arango DJ, Morton M, Gennari F, Kiplesund S, Contreras M, Watts C. Prevention of violence against women and girls: what does the evidence say? Lancet. 2014;385(9977):1555-66. http://dx.doi.org/10.1016/S0140-6736(14)61703-7.

7. World Health Assembly. Strengthening the role of the health system in addressing violence, in particular against women and girls, and against children. WHA67.15. https://afem.africa/wp-content/uploads/2017/10/World-Health-Assembly-Resolution-67.15.pdf (2014). Accessed Dec 11, 2020.

8. World Health Organization. Monitoring the building blocks of health systems: a handbook of indicators and their measurement strategies. https://www.who.int/healthinfo/systems/WHO_MBHSS_2010_full_web.pdf (2010). Accessed Dec 11, 2020.

9. Colombini M, Dockerty C, Mayhew SH. Barriers and Facilitators to Integrating Health Service Responses to Intimate Partner Violence in Low- and MiddleIncome Countries: A Comparative Health Systems and Service Analysis. Stud Fam Plann. 2017;48(2):179-200. doi:10.1111/sifp.12021.

10. Sardinha L, Nájera Catalán HE. Attitudes towards domestic violence in 49 low- and middle-income countries: A gendered analysis of prevalence and country-level correlates. PLoS One. 2018;13(10):e0206101. doi:10.1371/journal.pone.0206101.

11. Agrawal S, Banerjee. Perception of violence against women among future health professionals in an Industrial Township. Industrial Psychiatry Journal. 2010;19(3). 10.4103/0972-6748.90337.

12. Series on Violence Against Women. Lancet 2015. https://www.thelancet.com/series/violence-against-women-and-girls.

13. World Health Organization. Responding to intimate partner violence and sexual violence against women: WHO Clinical and Policy guidelines. https://www.who.int/reproductivehealth/publications/violence /9789241548595\$4en/ (2013).

14. National Institute of Population Research and Training - NIPORT/Bangladesh, Mitra and Associates/Bangladesh, and Macro International. Bangladesh Demographic and Health Survey 2007. 2009. Dhaka, Bangladesh: National Institute of Population Research and Training, Mitra and Associates, and Macro International.

15. National Institute of Population Research and Training - NIPORT/Bangladesh, Mitra and Associates, and ICF International. Bangladesh Demographic and Health Survey 2014. 2016. Dhaka, Bangladesh: NIPORT, Mitra and Associates, and ICF International.

16. Ministry of Health - MOH/Nepal, New ERA/Nepal, and ICF. Nepal Demographic and Health Survey 2016. 2017. Kathmandu, Nepal: MOH/Nepal, New ERA/Nepal, and ICF.

17. National Institute of Statistics of Rwanda, Ministry of Finance and Economic Planning/Rwanda, Ministry of Health/Rwanda, and ICF International Rwanda Demographic and Health Survey 2014-15. 2015. Kigali, Rwanda: National Institute of Statistics of Rwanda, Ministry of Finance and Economic Planning/Rwanda, Ministry of Health/Rwanda, and ICF International.

Page $9 / 11$ 
18. Bott S, Guedes A, Ruiz-Celis AP, Mendoza JA. Intimate partner violence in the Americas: A systematic review and reanalysis of national prevalence estimates. Rev Panam Salud Publica. 2019;43:e26. https://doi.org/10.26633/RPSP.2019.26

19. Department of Census and Statistics (DCS) and Ministry of Healthcare and Nutrition (MOH). Sri Lanka Demographic and Health Survey 2016.2018. Colombo, Sri Lanka: DCS and MOH.

20. Department of Census and Statistics (DCS) and Ministry of Healthcare and Nutrition (MOH). Sri Lanka Demographic and Health Survey 2006-07. 2009. Colombo, Sri Lanka: DCS and $\mathrm{MOH}$.

21. Newman C, Kabagwira A, Scislowicz J, Salentine S, Muramutsa F. Study on the readiness of the political, legal, health and community systems to respond to gender-based violence in three districts of the city of Kigali. https://www.intrahealth.org/sites/ihweb/files/attachmentfiles/GBV_assessment_report.pdf (2008). Accessed Dec 11, 2020.

22. Olson RM, García-Moreno C, Colombini M. The implementation and effectiveness of the one stop centre model for intimate partner and sexual violence in low- and middle-income countries: a systematic review of barriers and enablers. BMJ Glob Health. 2020;5(3): 10.1136/bmjgh-2019-001883.

23. Case Studies on Addressing VAW through Health System Response in LMICs. Supplementary Appendix to: García-Moreno C, Hegarty K, Lucas d'Oliveira AF, Koziol-MacLain J, Colombini M, Feder G. The health-system response to violence against women. Lancet. 2014. http://dx.doi.org/10.1016/S01406736(14)61837-7.

24. Online Education Course on Health Response to Violence against Women. https://www.unasus.gov.br/cursos/curso/45202. Accessed Dec 11, 2020.

25. Akhter H. The NGO Health Service Delivery Project (NHSDP) Final Report. 2018. Pathfinder International. Dhaka, Bangladesh.

26. Colombini M, Mayhew S, Hawkins B, Bista M. Agenda setting and framing of gender-based violence in Nepal: how it became a health issue. Health Policy and Planning: 2016. https://pubmed.ncbi.nlm.nih.gov/26412857/

27. Government of Nepal Ministry of Health and Population. Review of the scale-up, functionality and utilisation, including barriers to access, of One Stop Crisis Management Centres. 2020. Department of Health Services. Kathmandu, Nepal. http://www.nhssp.org.np/Resources/GESI/Scaling\%20Up\%200CMCs\%20\%20-\%20April\%202020.pdf.

28. Jhpiego. Strengthening Post Gender-Based Violence Health Services in Rwanda: Impact from a Quality Assurance Intervention. 2020. Draft.

29. Ministry of Health and Population with assistance from UNFPA and Jhpiego. Clinical Protocol on Gender Based Violence. 2015. Kathmandu, Nepal. http://reprolineplus.org/resources/clinical-protocol-gender-based-violence.

30. Health Sector Response to Gender Based Violence Standard Operating procedures for first contact point health care providers. Colombo, Sri Lanka. https://drive.google.com/file/d/1FQMM4chTk3AnFeDPkHoDDTAiXis4Mlsd/view (2019). Accessed Dec 11, 2020.

31. Strengthening health systems to respond to women subjected to intimate partner violence or sexual violence: a manual for health managers. Geneva: World Health Organization. https://apps.who.int/iris/bitstream/handle/10665/259489/9789241513005eng.pdf;jsessionid=C05B8D89DDB50FB6A4BDF876BAC03C86? sequence=1 (2017). Accessed Dec 11, 2020.

32. World Bank Group. Women, Business and the Law 2018. https://openknowledge.worldbank.org/bitstream/handle/10986/29498/9781464812521.pdf? sequence=10\&isAllowed=y (2018). Accessed Dec 11, 2020.

33. Government of the People's Republic of Bangladesh. Evaluation Sector. Impact Evaluation Study of Multi-Sectoral Programme on Violence against Women (2nd Phase). Implementation Monitoring and Evaluation Division, Ministry of Planning. https://imed.portal.gov.bd/sites/default/files/files/imed.portal.gov.bd/page/e773d5bf_182e_4fc5_a856_dfd3c8d05ced/women.pdf (2014). Accessed Dec 11, 2020.

34. Government of Nepal. Hospital Based One-stop Crisis Management Center (OCMC) Operational Manual http://www.nhssp.org.np/NHSSP_Archives/gesi/OCMC_Operational_manual_2067_2016.pdf (2016). Accessed Dec 112020.

35. Jayatilleke A, Yoshikawa K, Yasuoka, J. Training Sri Lankan public health midwives on intimate partner violence: a pre- and post-intervention study. BMC Public Health. 2015;331(15). https://doi.org/10.1186/s12889-015-1674-9.

36. Government of Nepal Population Division. Assessment of the performance of Hospital-Based One Stop Crisis Management Centres, Oct. https://un.info.np/Net/NeoDocs/View/3431 (2013).

37. Betron M, Thapa A, Amatya R, Thapa K, et al. Should female community health volunteers (FCHVs) facilitate a response to gender-based violence (GBV)? A mixed methods exploratory study in Mangalsen, Nepal. 2020. Global Public Health, 10.1080/17441692.2020.1839929.

38. BRAC Community Empowerment Programme. Snapshot of Programming on Violence Against Women. 2019. Dhaka, Bangladesh.

39. Government of Rwanda, Ministry of Health. Protocol on Multidisciplinary Treatment of Victims of Gender-based Violence and Child Abuse. 2015. Kigali, Rwanda.

40. Jejeebhoy S, Santhya K, Acharya R. Violence against women in South Asia: The need for the active engagement of the health sector. Global Public Health. 2014;9(6) 10.1080/17441692.2014.916736.

41. Majumdar A. Medical and Nursing Students' Knowledge and Attitudes Toward Violence Against Women in India. Education for Health. 2004: 3 10.1080/13576280400002627.

42. Jhpiego. Low Dose, High Frequency: A Learning Approach to Improve Health Workforce. Competence, Confidence, and Performance. 2016. https://hms.jhpiego.org/wp-content/uploads/2016/08/LDHF_briefer.pdf

43. Ministry of Health and Population, UNFPA and Jhpiego, Nepal. Health Response to Gender-Based Violence: Competency Based Training Package for Blended Learning and On-the-Job Training. Facilitators' Guide and Learners' Guide. 2016. https://nepal.unfpa.org/en/publications/health-responsegender-based-violence-facilitators-guide 
44. Government of Brazil. Secretariat for Policies for Women. Ordinance No. 04, 01.21.2015. Provides for the implantation of the Brazilian Women's House in the Municipality of Campo Grande, Mato Grosso do Sul.

45. Sohal A, Feder G, Barbosa E. Improving the healthcare response to domestic violence and abuse in primary care: protocol for a mixed method evaluation of the implementation of a complex intervention. BMC Public Health. 2018:971(18). https://doi.org/10.1186/s12889-018-5865-z

46. Human Rights Watch. Brazil's Empty Gestures Towards Women. https://www.hrw.org/news/2020/01/10/brazils-empty-gestures-women (2020). Accessed Dec 11, 2020.

47. Sohal A, Feder G, Boomla K, et al. Improving the healthcare response to domestic violence and abuse in UK primary care: interrupted time series evaluation of a system-level training and support programme. BMC Med. 2020): 18(48). https://doi.org/10.1186/s12916-020-1506-3.

48. World Economic Forum. Global Gender Gap Report. Geneva, Switzerland. http://www3.weforum.org/docs/WEF_GGGR_2020.pdf (2020). Accessed Dec 11, 2020.

49. World Economic Forum. Appendix B to Global Gender Gap Report. Geneva, Switzerland: https://reports.weforum.org/global-gender-gap-report2020/appendix-b-the-global-gender-gap-index-methodology-and-technical-notes/ (2020). Accessed Dec 11, 2020.

\section{Figures}

External referral to non-health sectors such as: Police, judiciary, women's groups, etc
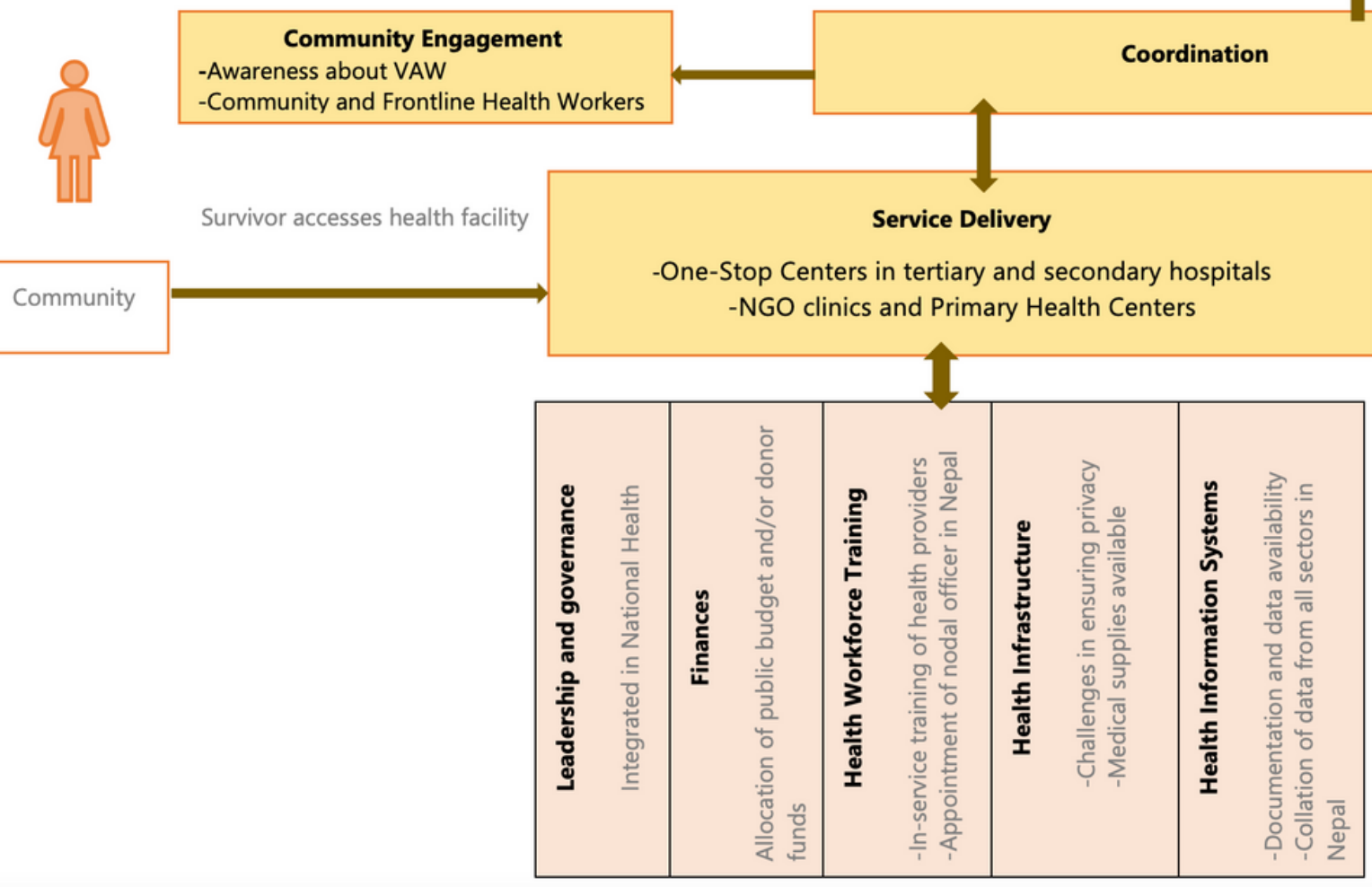

-One-Stop Centers in tertiary and secondary hospitals
-NGO clinics and Primary Health Centers

Community

Figure 1

Strengths and Gaps by Component of Health Systems Framework on Violence Against Women. VAW = violence against women; NGO = non-governmental organization.

\section{Supplementary Files}

This is a list of supplementary files associated with this preprint. Click to download.

- AdditionalFile1.docx 